\title{
Article
}

\section{The Confined Atom: James Clerk Maxwell on the Fundamental Particles and the Limits of Scientific Knowledge}

\author{
Charalampous, Charis
}

Available at http://clok.uclan.ac.uk/37395/

Charalampous, Charis (2021) The Confined Atom: James Clerk Maxwell on the Fundamental Particles and the Limits of Scientific Knowledge. Perspectives on Science, 29 (2). pp. 189-214. ISSN 1063-6145

It is advisable to refer to the publisher's version if you intend to cite from the work. http://dx.doi.org/10.1162/posc_a_00365

For more information about UCLan's research in this area go to http://www.uclan.ac.uk/researchgroups/ and search for < name of research Group>.

For information about Research generally at UCLan please go to http://www.uclan.ac.uk/research/

All outputs in CLoK are protected by Intellectual Property Rights law, including Copyright law. Copyright, IPR and Moral Rights for the works on this site are retained by the individual authors and/or other copyright owners. Terms and conditions for use of this material are defined in the policies page. 


\section{The Confined Atom: \\ James Clerk Maxwell on \\ the Fundamental Particles \\ and the Limits of \\ Scientific Knowledge}

Charis Charalampous

UCLan Cyprus

This paper distinguishes in Maxwell's thought between "atomic molecules" and "ultimate atoms," and arrives at a set of properties that characterize each type of atom. It concludes that Maxwell is a mathematical atomist, an approach that entails the notion that although it is impossible to observe the ultimate atoms as free particles, we can nevertheless study them as mathematical observables, on the caveat that mathematical formalism remains tied to phenomenalism and to theoretical interpretations of such phenomena as, for example, mass and force variations, gravitational pull, gas diffusion and viscosity, and heat conduction.

Each generation, from the earliest dawn of science to the present time, has contributed a due proportion of its ablest intellects to the quest of the ultimate atom. (Maxwell 1873, p. 437)

\section{Introduction}

Nineteenth-century physicists and philosophers inherited from their predecessors a number of atomic explanations, which they developed and/or subjected to criticism in varied ways. In David M. Knight's words, "the nineteenth century stands out as a century in which scientists were deeply divided over atomic explanations. There was no one classical, received atomic theory, but rather a number of theories overlapping in their explanatory ranges" (Knight 1967, p. 2). To provide a sketchy overview of these theories, mathematical physicists made use of atoms principally in the sense of inelastic corpuscles or inertial force points, often characterized, as per Boscovich and Kant, by various attractiverepulsive forces acting either inherently from within the corpuscles/energy points or lying in the medium between the particles. The physics of Newton, the Carnots, Lagrange and Poisson was based on the existence of indivisible hard bodies acting mechanically in conservative systems, both ponderable 
matter and the imponderable ether being presumed to be composed of such bodies. The chemists' idea of atoms was essentially Daltonian inasmuch as they identified undecomposed chemical elements with specific elementary atoms of differing and distinct weights (Nye 1976, p. 247). But as Mary Jo Nye has observed, "this conception had more affinity with the Aristotelian-Averroestic minima naturalia than with the qualitatively identical Greek-Democritean atoms, although like Democritus' atoms, Dalton's were physically and chemically indivisible" (1976, p. 247). Not only did nineteenth-century physicists rework received theories of the atom, but they developed new explanatory scenarios, one of the most famous ones being Kelvin's vortex atom, for which Maxwell himself had expressed a degree of admiration. Here I argue that Maxwell, too, promoted a novel view of the atom, one that was rooted in kinetic theory: inherent in Maxwell's writings is the theory of mathematical atomism, according to which the fundamental building blocks of physical reality, what he refers to as "ultimate atoms," cannot exist in nature in an unconfined state. It would therefore be impossible to observe them empirically as free particles, but they can be studied mathematically. This is a modest essay in that very problematic, placing a particular focus on Maxwell's encyclopedia entry of 1875 and on other of his works from the 1870s, which are examples of his more mature thinking on atoms and molecules.

The distinction between "atomic molecules" and "ultimate atoms" has been anticipated in extant criticism in varied ways, and so this essay brings conceptual sharpness to a distinction that is latent within the literature. A focus on this distinction allows for the study of the relation in Maxwell's thought between the two types of atoms, which in turn shows that for him the fundamental building blocks of all physical reality remain in a confined state never to be empirically observed, although we can study them via a fruitful combination of phenomenalism and mathematical formalism. Maxwell's methodology entailed the premise that any description of these ever elusive particles must account for spectroscopic observations (i.e., vibrational/rotational motions) and theoretical interpretations of such phenomena as, for example, mass and force variations, gravitational pull, gas diffusion and viscosity, and heat conduction. The need for any atomic theory to satisfy these demands was Maxwell's scientific compass when he came to critically assess some of the period's most influential atomic theories. It was also the principle that led him to assert the existence of atomic molecules on the one hand and ultimate atoms on the other as the constituent components of the former type of atoms.

The nineteenth century, as Peter Achinstein put it, saw the "atom's empirical eve," and Maxwell contributed substantially to the debate of as to whether bodies consist of continua or indivisible parts (Achinstein 2007; see also Garber 1978). This question stirred spirited debates among the period's scientists and philosophers (i.e., Knight 1967; Scott 1970; Levere 1971; Nye 1976; and 
Rocke 1984). As Maxwell notes, "the question of the existence of atoms has once more become conspicuous among scientific inquiries" (Maxwell 1875a, p. 36). Maxwell's preoccupation with the atomic constitution of bodies began at least as early as in 1849, when he was only eighteen years old and an undergraduate at Edinburgh University (Maxwell 1990, I, 1849-1850, pp. 133-83). He returned to the problem when he was still revising his paper on Saturn's rings in 1857, at which time he was already professor of natural philosophy at Marischal College, Aberdeen, and elected fellow of the Royal Society of Edinburgh (Maxwell 1990, I, 1857, pp. 553-56). But his more serious engagement with the question coincides with his work on the kinetic theory of gases, which led him to a study of the collisions of particles as a means of establishing the properties of gases (Maxwell 1990, I, 1859, pp. 606-11). ${ }^{1}$ On May 10, 1859, just a few months before he read his first paper on the subject at the Meeting of the British Association for the Advancement of Science at Aberdeen, Maxwell wrote a letter to George Stokes with the following question: "do you think there is any so complete a refutation of this theory of gases as would make it absurd to investigate it further so as to found arguments upon measurements of strictly 'molecular' quantities before we know whether there be any molecules?" (Maxwell 1990, I, 1859, p. 610). Despite the degree of skepticism that Maxwell expresses here about the validity of the theory of the molecular structure of bodies, his exercises suggested to him that only the existence of a "complete refutation" of this theory could thwart his efforts. This somewhat agnostic attitude gradually gave way to a greater degree of certainty, as this is expressed his seminal paper of 1867 "On the Dynamical Theory of Gases" (Read on May $31,1866)$, where he abandons his former Clausian hypothesis that molecules are hard elastic spheres, now supposing them, in a Boscovichian twist of thought, to repel each other with forces varying inversely as the fifth power of the distance (Maxwell 1867, p. 51). ${ }^{2}$ Here, Maxwell evokes Democritus, Epicurus and Lucretius, and frames his inquiry as a problem that inheres in the question of as to whether matter is a continuum or composed of discreet and indivisible particles. Quite suggestively, the paper opens with the following statement: "Theories of the constitution of bodies suppose them either to be continuous and homogeneous, or to be composed of a finite number of distinct particles or molecules" (Maxwell 1867, p. 49). Theories that uphold the notion of a continuum lack solid mathematical foundations capable of describing the observed properties of bodies, and they are therefore to be seen as dogmas rather than

1. On the history of kinetic theory and Maxwell's role in it, see Brush 1976; Porter 1981, pp. 77-116; Porter 1986, pp. 111-26; Garber, Brush and Everitt 1986, esp. pp. 4-20; and Brush and Garber, 1995.

2. For more information on the reception of the Boscovichian atom by Maxwell and other nineteenth-century theorists, including Kelvin, see Heilbron 2015, pp. 99-116. 
theories of substantial scientific merit (Maxwell 1867, p. 49). As he states in a lecture he delivered before the British Association at Bradford in 1873, "We should thus, in imagination, arrive at the atom, which, as its name literally signifies, cannot be cut in two. This is the atomic doctrine of Democritus, Epicurus, and Lucretius, and, I may add, of your lecturer" (Maxwell 1873, p. 47). Maxwell's conviction in the atomic constitution of bodies is repeated in various places throughout his mature works, such as in a chapter in his popular Theory of Heat (1871) entitled "Molecular Theory of the Constitution of Bodies" (Maxwell 1871a, pp. 281-312), and in 1875 in a two-part paper in Nature entitled "On the Dynamical Evidence of the Molecular Constitution of Bodies." Here, he assertively notes that "whatever may be our ultimate conclusions as to molecules and atoms, we have experimental proof that bodies may be divided into parts so small that we cannot perceive them" (Maxwell 1875b, p. 357). Our understanding of the nature of the "molecules and atoms" may still be inconclusive, but Maxwell is adamant in his conviction that bodies are composed of atoms. It is therefore natural to assume that Maxwell evolved into a firm supporter of atomism. But he was not merely motivated to find an answer to the question of as to whether matter is composed of atoms or not. His interest was tied with the issue of the ontology and properties of the fundamental particles of physical reality.

\section{Maxwell's Ultimate Atoms and Atomic Molecules}

The distinction between "atoms" and "molecules" was at least as old as Clausius' seminal paper of 1857, where, as Elizabeth Garber has observed, he "distinguished atoms from molecules, and he demonstrated that molecules were complex bodies, subject to rotary as well as translational motions" (Garber 2014, pp. 140-41). And as David M. Knight noted, "Maxwell used 'molecule' wherever possible, defining the term as the smallest possible portion of a particular substance, while atoms were particles which could not be cut in two" (Knight 1967, p. 77). Following Clausius, Maxwell did distinguish between atoms and molecules, but this distinction is not as clear-cut as we might often assume, because in Maxwell's mind, "molecules," although they admittedly have a complex internal structure, they may very well be physically indivisible, viz., atomic. Thus, Maxwell wrote to the Bishop of Gloucester and Bristol Charles John Ellicott in November 1876 that "The comparison of atoms or of molecules to 'manufactured articles', was originally made by Sir J. E D. Herschel” (Maxwell 2002a, vol. III, 1876, Part I, p. 416). In this example, Maxwell's "or" is an inclusive locution, like the Latin "vel.," to signify that if molecules, although having an internal structure, are indeed indivisible and indestructible (atomic), then God manufactured them as they are at some point in time. On such occasions, "molecules" refers to indivisible 
particles, so that "atoms" and "molecules" can be read as cognates. In his lecture on "Molecules" of 1873, Maxwell explained that

A drop of water, to return to our former example, may be divided into a certain number, and no more, of portions similar to each other. Each of these the modern chemist calls a molecule of water. But it is by no means an atom, for it contains two different substances, oxygen and hydrogen, and by a certain process the molecule may be actually divided into two parts, one consisting of oxygen and the other of hydrogen.

According to the received doctrine, in each molecule of water there are two molecules of hydrogen and one of oxygen. Whether these are or are not ultimate atoms I shall not attempt to decide. We now see what a molecule is; as distinguished from an atom [...] An atom, if there is such a thing, must be a molecule of an elementary substance. Since, therefore, every molecule is not an atom, but every atom is a molecule, I shall use the word molecule as the more general term. (Maxwell 1873, p. 437)

It is quite straightforward to understand why a molecule of water cannot be termed an atom. Naturally, then, "every molecule is not atom." However, Maxwell's "but every atom is a molecule" may read somewhat problematic: in what sense is every atom a molecule and how are they to be distinguished from the particles to which he refers in the same passage as "ultimate atoms"? As indicated above, there are two types of molecules in Maxwell's thought, viz., molecules that are known to be physically divisible into constituent parts, like a water molecule, and molecules that although may consist of parts, they are nevertheless physically indivisible. We may refer to the latter as "atomic molecules." Indeed, in his 1875 entry for Encyclopcedia Britannica, Maxwell asserted that a molecule can be treated as an atom only insofar as it operates as a conservative system: "Our definition of a molecule is purely dynamical. A molecule is that minute portion of a substance which moves about as a whole, so that its parts, if it has any, do not part company during the motion of agitation of the gas" (Maxwell 1875a, p. 40). For Maxwell, molecules can be said to be atomic on the caveat that they travel as indivisible quantities, but the fact that they are physically indivisible should not inexorably lead to the conclusion that they do not have internal constituent components, viz., "ultimate atoms." We may therefore distinguish from the outset between "atomic molecules," "molecules" and "ultimate atoms." The next sub-sections concentrate on Maxwell's interpretation of the properties and nature of these two types of atoms, before turning to his mathematical atomism and the ways in which it underpins his conviction that although an answer to the problem of the atoms' origin is scientifically untenable, we can nevertheless describe their structure and properties mathematically. 


\subsection{Ultimate Atoms}

Maxwell opens his encyclopedia article with a brief overview of ancient Greek theories of matter from Anaxagoras and Democritus to Epicurus, and he swiftly leaps from the Greeks to Descartes and Leibniz, before landing at a rather more extensive account of Boscovich's atomism. He regards the Boscovichian atom an example of pure monadism, whereby we have elements that occupy space by means of force rather than material extension. Unlike Kant, who theorized that monads emit two overlaid forces (the attractive and the repulsive), Boscovich conceptualized his puncta as force-shell atoms projecting one unified force that repels at some distances and attracts at others (Boscovich [1758] 1966, p. 10). ${ }^{3}$ Boscovich took into consideration contemporary developments in chemistry and theorized that the degrees of matter solidity we observe, from rigid bodies to liquids and gases, are rooted in the ways that atoms cohese. He first developed his theory of the limits of cohesion and unconhesion in relation to the repulsive/attractive force radiating out of an atom in his De Lumine (1748), where he argued that the elementary particles are indivisible and materially unextended puncta that occupy space by means of the repulsive force they exert around them (Boscovich 1766, p. 68). Boscovich's atoms do not touch, like the atomist's theory or Leibniz's monads (Boscovich 1766, p. 68). He rejected the arguments for infinite divisibility, which are based on a mathematical and geometrical analysis of continua, and held that his theory picks no argument with geometricians and mathematicians while retaining the atom's indivisibility (Boscovich 1766, pp. 70-72).

Each of Boscovich's atoms, Maxwell summarizes, "is an indivisible point, having position in space, capable of motion in a continuous path, and possessing a certain mass, whereby a certain amount of force is required to produce a given change of motion. Besides this the atom is endowed with potential force, that is to say, that any two atoms attract or repel each other with a force depending on their distance apart" (Maxwell 1875a, p. 37). An ultimate atom, or what he alternatively referred to as "the pure elementary atom" (Maxwell 2002a, vol. III, 1874, Part II, p. 119), should have these Boscovichian properties indeed: a stated and conserved mass along with the tendency to preserve its state of rest or uniform motion, and an attractive/repulsive force. As Maxwell notes in 1871 in his Theory of Heat, even "If a molecule were a mathematical point," it would still be "endowed with inertia and with attractive and repulsive forces $[\ldots]$ But if it be a body having parts and magnitude, these parts may have motions of rotation or of vibration relative to each other, independent of the motion of the centre of gravity of the molecule" (Maxwell 1871a, p. 290).

3. For a concise account of Boscovich's theory of the wave-like behaviour of the repulsiveattractive force that the puncta radiate, a factor that sets the Ragusan philosopher's theory apart from Kant's, see Holden 2004, esp. p. 247. 
That said, pace Boscovich, Maxwell follows Faraday's suggestion that atoms may be mutually penetrable and argues that "Boscovich himself, in order to obviate the possibility of two atoms ever being in the same place, asserts that the ultimate force is a repulsion which increases without limit as the distance diminishes without limit, so that two atoms can never coincide" (Maxwell 1875a, p. 37). For Maxwell, "this seems an unwarrantable concession to the vulgar opinion that two bodies cannot co-exist in the same place. This opinion is deduced from our experience of the behavior of bodies of sensible size, but we have no experimental evidence that two atoms may not sometimes coincide" (Maxwell 1875a, p. 37). Maxwell insists that we should not apply our presumptions about the nature of matter to atoms. In this way, "Many persons cannot get rid of the opinion that all matter is extended in length, breadth, and depth. This is a prejudice [...] arising from our experience of bodies consisting of immense multitudes of atoms" (Maxwell 1875a, p. 37). Maxwell contends that Boscovich's account still suffers from the perceived notion that two elements cannot occupy the same unit of space at the same time (see also Harman 1998, pp. 184-86). This assumption should be seen as a dogma unsupported by scientific evidence. ${ }^{4}$ We may thus deduce that a third property of Maxwell's ultimate atoms is that two or more such particles may interpenetrate and occupy the same point in space. The property of atomic interpenetrability is predicated in Maxwell's mind on the assumption that within the repulsive force field there exists an attractive region despite the very close proximity of the particles (Maxwell [1876] 2002a, vol. III, Part I, p. 348).

To sum up, we can identify three properties that characterize Maxwell's ultimate atoms. They have a conserved mass along with the tendency to preserve their state of rest or uniform motion, they exert attractive and repulsive forces, and they may very well be capable of occupying the same point in space. As we will see in the third section, we may also add that Maxwell's ultimate atoms do not exist in nature as unconfined, free particles and that they can thus be studied only mathematically. However, in order to appreciate the force and logic of Maxwell's view, it is instructive to explore first his interpretation of the properties of atomic molecules.

\subsection{Atomic Molecules}

Maxwell refrained from drawing any assertive conclusion regarding the question of as to whether molecules are extended or unextended, material bodies or the centers of force fields. They must nevertheless have a stated mass and exist in a process of continuous reconfigurations determined by their motions (Maxwell 1875a, p. 38). According to Maxwell, by calculating a molecule's mass in relation to "the amount of motion which exists among the small parts,

4. For an earlier exposition of this thesis, see Maxwell 1990, vol. I, 1866, p. 434. 
independent of the visible motion of the medium as a whole," one should be able to determine the total energy of a material system. Regardless of as to whether a material system has a particular velocity or not, its internal parts have kinetic energy, the calculation of which will give us information about the relative masses of the molecules considered as moving bodies (Maxwell 1875 a, p. 38). In order to determine a molecule's kinetic energy, Maxwell considers material systems in states of stationary motion and translational speed. The kinetic energy of the parts of the former system is the outcome of the attractive and repulsive forces that each particle exerts on the other. This naturally leads to a state of equilibrium where we have a material system in stationary motion, the velocity and direction of its parts remaining unchanged. In both cases, particles are to be seen as conservative systems in line with the law of the conservation of energy. This is self-evident because "all bodies with which we are acquainted are conservative systems, which would not be the case unless their parts were also conservative" (Maxwell 1875a, p. 38).

Once Maxwell began to calculate the masses of molecules and to describe their motions, it became obvious to him that they must have a complex internal structure, primarily because of their vibrational motions as they absorb and emit light at various frequencies. The viscosity of gases played an important role in the historical development of the kinetic theory of gases (Darrigol and Renn 2013, pp. 765-88). In 1860, Maxwell had proposed that gases possess a distribution of velocities. His basic hypothesis is that the numerous collisions between molecules in a gas would produce a statistical distribution of velocities in which all velocities might occur, with a known probability. But Maxwell was troubled because his theorem had "the curious result" that viscosity is independent of pressure, which was "certainly very unexpected" (Maxwell 1990, vol. I, 1859, p. 610). Maxwell made the first reliable measurements of gas viscosities in order to determine the dependence of gas viscosity on temperature and pressure. The results were reported in 1866, reconciling his kinetic theory of gases with observed gas viscosities. He found that the viscosity of air at a given temperature remains constant when the pressure varies between 1/2 and 30 inches (Maxwell 1867, pp. 49-88).

Despite the success of his formulas, Maxwell was never able to reconcile his kinetic theory of gases with the observed ratio of specific heats for diatomic gases. One could use the Maxwell-Boltzmann distribution of molecular speeds to determine the average kinetic energy of a particle in a gas, and show that it agrees with the equipartition theorem, which states that energy is shared equally amongst all energetically accessible degrees of freedom of a system. But the problem rested in the fact that the expected ratio of specific heats in the case of diatomic molecules, such as hydrogen, nitrogen or oxygen, was higher than the ratio yielded by experiments. In 1857 Clausius had derived a general formula for the ratio of specific heats, from which it follows 
that this ratio should be equal to $5 / 3$ for a monatomic gas regarded as a system of elastic spheres. The spectroscope, nevertheless, was revealing that all molecules, even monatomic ones, cannot be hard elastic bodies and that they must have a complex internal structure. If the parts of a molecule can move in such a way as to absorb and emit light at various frequencies, then they should also, according to kinetic theory, have their proper share of the total energy of the molecule (Maxwell 1875a, pp. 42-3). This is because it is possible to account for this observed molecular vibration only by assuming the existence of an internal motion or interaction: "In fact one of the first conditions which a molecule must fulfil is, apparently, inconsistent with its being a single hard body. We know from those spectroscopic researches which have thrown so much light on different branches of science, that a molecule can be set into a state of internal vibration" (Maxwell [1870] 1871b, p. 6). If molecules can rotate or vibrate, the ratio should be smaller than $5 / 3$. As Stephen G. Brush has noted here, "the general formula for the ratio is $\gamma=1+\frac{2}{3+n}$, where $n$ is the number of degrees of freedom of the internal motions of the molecule. According to Newtonian mechanics, a system of molecules will have 3 translational degrees of freedom, corresponding to the three independent directions in space in which each particle may move" (Brush 2003, p. 28). The rest degrees of freedom represent internal degrees of freedom $(n)$. According to the general formula, a diatomic molecule, regarded as a mechanical system of two spheres held together by some kind of attractive forces, would be expected to have at least $n=3$ and therefore $\gamma=4 / 3$. Although later experiments showed that the ratio of specific heats of monatomic gases were in agreement with the formula for no internal degrees of freedom, diatomic molecules yielded a ratio of $\gamma=7 / 5$ and $n=2$. Each mode of translation or rotation corresponds to a single degree of freedom because only kinetic energy is present. But any mode of vibration corresponds to two degrees of freedom because there are two stores of energy: potential and kinetic. As a result, the total number of degrees of freedom is predicted to be 7 , the sum of 3 for the translational degrees of freedom, 2 for the rotational ones, and 2 for the single vibrational mode. However, as has already been stated, the value of $\gamma$ for air, which consists largely of the diatomic molecules of oxygen and nitrogen, is 1.408 at usual temperatures, which is very close to $7 / 5$. This suggests that for diatomic molecules, $n$ is 5 rather than 7. Maxwell could not find any convincing reason why the equipartition theorem should not apply to the internal motions of gas molecules. ${ }^{5}$ The discrepancy between theoretical and experimental specific heats remained an unsolved problem until the advent of quantum theory.

5. For an illuminating discussion of the limitations of the kinetic theory for establishing a molecular science, see Garber 1978, pp. 265-97. 
The incompleteness of the kinetic theory of gases notwithstanding, contemporary advances convinced Maxwell that molecules should not be presumed to be material bodies, but we may assume them to be conservative systems with a stated mass and in continuous motion. They radiate a repulsive/attractive force and they are capable of vibration, emitting and absorbing light at varying frequencies, which is indicative of the fact that they must have a complex internal structure. Maxwell, moreover, suggests that we may limit the essential conditions that an atomic molecule must satisfy to three: "permanence in magnitude, capability of internal motion or vibration, and a sufficient amount of possible characteristics to account for the difference between atoms of different kinds" (Maxwell 1875a, p. 45). The first condition is a restatement of the principle of an atomic molecule's energy conservation inasmuch as since we are seeking a stable and indivisible particle, then it must have a stated and conserved mass. The second condition reminds us that atomic molecules must be capable of vibratory or rotational motion, and the third one holds that in order to be able to account for the diversity of substances we may observe in the natural world, atomic molecules cannot be homogeneous. We may also add that, as in the case of the ultimate atoms, we should not suppose that atomic molecules are impenetrable. Like ultimate atoms, then, atomic molecules could have the property of inter-penetrability. If this were the case, then there must exist some attractive forces within the region of the repulsive force capable of binding the two into a single point in space (Maxwell 2002a, vol. III, 1876, Part I, p. 348).

\section{Maxwell's Evaluation of Atomic Theories}

Maxwell applied his concepts of atoms and molecules to contemporary atomic theories. Having provided the method for determining a molecule's mass and motion in his encyclopedia article, he revisits what he appears to have conceived of as the main families of atomism: the Democritean solid atoms, the Boscovichian force centers and Thomson's vortex atoms. The Democritean atom, which according to Maxwell was also adopted by Newton, satisfies the condition of establishing the permanent properties of bodies, but it fails to account for the vibrations of molecules that the spectroscope revealed. The mathematical investigations of Poisson, Navier and the French School were premised on the assumption that bodies are composed of atoms whose configurations determine those objects' properties, but for Maxwell, "as the same results have been obtained by Stokes and others without any such hypothesis, this of itself is no evidence of atomic structure" (Maxwell [1874] 2002a, vol. III, 1874, Part I, p. 125). Boscovich's force centres may be truly indivisible and have more merit for the mathematician who has no problems introducing new forces into equations. However, a single structure-less force atom has no intrinsic vibration. Only an interaction between two or more 
such force centers could yield vibrations (see also Nye 1976, p. 254). Boscovich's free puncta, therefore, introduce the possibility of separation and instability, given that we imagine molecules consisting of many such centers whose interactions cause the system's vibrational/rotational motions: "Such centres of force are no doubt in their own nature indivisible, but then they are also, singly, incapable of vibration. To obtain vibrations we must imagine molecules consisting of many such centres, but, in so doing, the possibility of these centres being separated altogether is again introduced" (Maxwell 1875a, p. 45). The fact that ultimate atoms have a stated mass and an attractive/repulsive force may indeed appear to bring Boscovich's puncta and Maxwell's ultimate atoms to a close epistemological proximity. After all, as Maxwell asserted in 1877 in his review of Henry William Watson's Kinetic Theory of Gases, "the best thing we can do is to get rid of the rigid nucleus altogether, and substitute for it an atom or Boscovich - a mathematical point endowed with mass and with powers of acting at a distance on other atoms" (Maxwell 2002b, vol. III, 1877, Part II, p. 499). And as he wrote in 1879 to Otto Schmitz-Dumont, just a few months before passing away, "If we are to give up a 'continuum', let us try what we can do with the force-points of Boscovich, each of which has a determinate mass" (Maxwell 2002b, vol. III, 1879, Part II, p. 736). But Maxwell's statements about Boscovich's atom theory are about exploring the contours of such a theory rather than endorsing it. Maxwell holds that Boscovich erroneously assumed his force-shell atoms to be impenetrable, but most importantly, puncta exist in nature as free particles, thereby introducing the possibility of instability.

According to Maxwell, "the vortex ring of Helmholtz, imagined as the true form of the atom by Thomson, satisfies more of the conditions than any atom hitherto imagined" (Maxwell 1875a, p. 45). Thomson's atom is an elastic and indivisible energy ring in permanent motion. He was struck by the evident of permanence, indivisibility and properties of Helmholtz's "water twists," which were vividly and dramatically demonstrated by Tait's smoke rings. Tait's presentations showed that smoke rings behave as independent solids, which on collision rebound, exhibiting elasticity. Smoke rings also exhibit vibrational modes in their circular form, and each attempt to cut the smoke rings with a knife results in the rings simply wriggling around the knife, suggesting a form of indivisibility that does not rely on density, solidity or force, but on the ring's intrinsic property of motion. Helmholtz's vortex theory and Tait's illustrations inspired Thomson's vortex theory of the atom, according to which atoms are knotted and linked tubular vortices, loci of a special type of rotary motion within the ether. On this view, matter becomes a mode of motion. Thomson developed the mathematical basis of his theory, enlisting in its behalf the support of many distinguished colleagues. What Maxwell found appealing in Thomson's theory is the conception of matter as a mode of 
motion, which does not uphold a sharp distinction between matter and energy. And unlike Boscovich's puncta, Thomson's atom is penetrable and it can vibrate on its own. It is therefore quantitatively and qualitatively permanent, it vibrates, it is elastic, and it has axiomatic simplicity (Maxwell 1875a, p. 45).

Although the vortex atom satisfied most of Maxwell's conditions (i.e., permanence, penetrability, vibration), it was nevertheless an incomplete theory with fundamental difficulties. ${ }^{6}$ For Maxwell, "the glory of surmounting" these problems "would be unique." Firstly, the mathematics that describe the motions of Thomson's fluid is lacking (Maxwell 1875a, p. 45). But most importantly, Thomson's "primitive fluid has no other properties than inertia, invariable density, and perfect mobility" (Maxwell 1875a, p. 45). These properties do not agree with the theoretical interpretations of mass, gravitational pull between particles and heat conduction (Harman 1998, pp. 197-98). It would seem absurd, Maxwell continues, to seek an explanation for mass because it is integral to the very essence of matter, and Thomson does attribute mass to his vortex rings. However, its application to material bodies is problematic. On Thomson's theory, one must explain the inertia of what is merely a mode of motion. According to Maxwell, then, "It is true that a vortex ring at any given instant has a definite momentum and a definite energy, but to shew that bodies built up of vortex rings would have such momentum and energy as we know them to have is, in the present state of the theory, a very difficult task" (Maxwell 1875a, p. 45). Being perfectly elastic, moreover, vortex atoms fail to account for the attraction between atoms. If atoms rebound against each other and fly off with the same velocity with which they started before impact, there will be no decrease in their velocities to account for attraction. If, on the other hand, particles rebound with slower velocities, attraction between bodies would be produced. Thomson published a paper addressing this problem. Here, as Maxwell notes, he "suggests that they are vortex atoms, which are set into a state of vibration at impact, and go off with a smaller velocity of translation, but in a state of violent vibration" (Maxwell 1875a, p. 47). However, this solution introduced a new set of problems: the rotational or vibrational motion of vortex atoms can now be explained only if they are conceived of as inelastic "material systems," not as "mere points" (Maxwell 1875a, p. 47), thereby losing aspects which Maxwell found appealing in Kelvin's theory, viz., their ability to vibrate on their own, their axiomatic simplicity and elasticity. This is not to imply that Maxwell discerned any fatal flaws in Thomson's theory, but that it was still unfinished, with serious difficulties yet to overcome.

6. For more information on the merits and problems of Thomson's vortex atomic theory, see Smith and Wise 1989, pp. 417-44. For a full account of the vortex atom theory and Maxwell's view on it, see also Kragh 2003. 


\section{Maxwell's Mathematical Atomism}

Given Maxwell's overall account, the ability of atomic molecules to vibrate seems to introduce a fundamental contradiction. If an atomic molecule is supposed "to execute vibrations of different periods, as we know that molecules do," then they should have a complex internal structure (Maxwell 1875a, p. 45). A molecule cannot therefore be a material point: "we learn from the spectroscope that a molecule can execute vibrations of constant period. It cannot therefore be a mere material point, but a system capable of changing its form" (Maxwell 1875b, p. 375). And yet, Maxwell is adamant in his Democritean conviction that "the molecule is incapable of growth or decay, of generation or destruction $[\ldots]$ the molecules out of which these systems are built - the foundation stones of the material universe-remain unbroken and unworn" (Maxwell 1873, p. 441). As Freeman Dyson has noted in his account of Maxwell's presidential Address in Liverpool (1870), "Maxwell explained how the ancient theory that matter is composed of atoms ran into a logical paradox. On the one hand, atoms were supposed to be hard, impenetrable and indestructible. On the other hand, the evidence from spectroscopy and chemistry showed that atoms have internal structure and are influenced by outside forces" (Dyson 1999). For Maxwell, nevertheless, atomic molecules are divisible only conceptually and mathematically. In the second part of "On the Dynamical Evidence of the Molecular Constitution of Bodies" (1875), for example, he notes that "we suppose that the constituents of a molecule are atoms, and that each atom is what is called a material point" (Maxwell 1875c, p. 375). Such particles are "incapable of rotatory energy or internal motion" (Maxwell 1875c, p. 375). On Maxwell's theory, gas molecules, like any other molecule, must be capable of vibration, a property which can be explained only if these molecules consist of points characterized by inertia, a radiating force that can repel or attract neighboring atoms and, possibly, penetrability. But the fact that atomic molecules are to be seen as "unbroken" and conservative systems means that ultimate atoms cannot exist in an unconfined state, an atomic molecule remaining only mathematically divisible into its constituent parts. ${ }^{7}$

7. It would be interesting to touch lightly here upon the relation between Maxwell's ultimate atoms and quarks, if only to indicate that our modern physics of particles have taken up this idea. As Murray Gell-Mann argued ever since he first proposed their existence, quarks can be observed only as bound particles and mathematical entities, not as free physical objects. For Gell-Mann, the physicists' attempts to observe the quarks were thus bound to fail. In Gell-Mann's own words, "up to the present, numerous authors keep stating or implying that when I wrote that quarks were likely to be 'mathematical' and unlikely to be 'real', I meant that they somehow weren't there. Of course I meant nothing of the kind [...] I have always believed instead in mathematical quarks, ones that do not emerge singly to be observed or utilized" (Gell-Mann 1997, pp. 626-27). 
A number of commentators have drawn attention to the notion that, for Maxwell, no atomic theory will ever be formidable enough to reveal the mechanics of existence, humbly conceding the limitations of scientific knowledge and even of human imagination itself (Tyndall 1871, p. 83; Pullman 1998, pp. 154-56; Marston 2007, pp. 731-40; Pratt-Smith 2014, pp. 233-57; Marston 2014, pp. 258-91). To use P. M. Harman's account as an example, it is often argued that,

Following John Herschel in his Preliminary Discourse on the Study of Natural Philosophy (1830), Maxwell considered that molecules had "the essential character of a manufactured article," and argued that the exact similarity of each molecule to all others of the same kind "precludes the idea of its being eternal and self-existent" [...] The similarity of molecules was an argument for their creation, the molecules remaining to this day as they were created, and "the ineffaceable characters impressed on them" were "essential constituents of Him who in the beginning created, not only the heaven and the earth, but the materials of which heaven and earth consist." No natural process could affect the nature of the immutable molecules, and "we are therefore unable to ascribe either the existence of the molecules or the identity of their properties to the operation of any causes which we call natural." The basis of his argument that molecules were immutable, then, was to be found in the belief that their similarity was outside the boundaries of science. (Heimann [sic] 1970, p. 203)

The atom's properties may remain "ineffaceable" in terms of their ultimate origin, but we do have the ability to study them. As Maxwell writes in "Molecules," just after expressing his agreement with Herschel's thesis on the manufactured article,

Thus we have been led, along a strictly scientific path, very near to the point at which Science must stop. Not that Science is debarred from studying the internal mechanism of a molecule which she cannot take to pieces, any more than from investigating an organism which she cannot put together. But in tracing back the history of matter Science is arrested when she assures herself, on the one hand, that the molecule has been made, and on the other, that it has not been made by any of the processes we call natural. (Maxwell 1873, p. 441)

The central thesis of Maxwell's argument here is that because of "the exact equality of each molecule to all others of the same kind," it is impossible to conceive of how atomic molecules could have come into existence through a natural process. Spontaneous ex nibilo creation being an unnatural process as well, atomic molecules must have been manufactured as they are at some point 
in time by an intervening agent. ${ }^{8}$ Maxwell also insists that it is via "a strictly scientific path" that we are led "very near to" the conclusion that an understanding of the nature of the fundamental building blocks of physical reality lays beyond the reach of science. The fact that atomic molecules are indivisible and that a scientific answer as to their creation is untenable does not necessarily mean that science is altogether unable to study their "internal mechanism." To recall Maxwell's words, science is not "debarred from studying the internal mechanism of a molecule which she cannot take to pieces." To begin with, we have the spectroscope, which can yield some information on the structural properties of atomic molecules (Maxwell 1873, p. 440). But perhaps more importantly, we have mathematics. As a point of illustration, we may turn to Maxwell's Theory of Heat. In a sub-chapter titled "Kinetic Energy of a Molecule," Maxwell notes that the mean kinetic energy of agitation of a molecule is the product of its mass by half the mean square of its velocity. However, he continues to observe that "This is the energy due to the motion of the molecule as a whole, but its parts may be in a state of relative motion. If we assume, with Clausius, that the energy due to this internal motion of the parts of the molecule tends towards a value having a constant ratio to the energy of agitation, the whole energy will be proportional to the energy of agitation" (Maxwell 1871b, p. 297. See also, Maxwell 1871b, p. 306; Maxwell 1995, vol. II, 1873, pp. 959-60; and Maxwell 1875c, p. 375). Maxwell is combining phenomenalism and mathematical formalism in order to arrive at a working hypothetical idea about the behavior of an atomic molecule's constituent components which is not unwarranted. Here, we may identify yet another probably Herschelian influence on Maxwell's thought. As P. M. Harman has observed, "Herschel emphasized the role of mathematics as providing the key to scientific knowledge, and he placed dynamics, the science of force and motion, 'at the head of all the sciences"' (Harman 1988, p. 85). Maxwell, too, Harman notes elsewhere, "stressed the importance of the link between the mathematical formalism of dynamics and the physical reality depicted [...] it was his aim to translate the mathematical symbols 'from the language of the calculus into the language of dynamics', so that this language should express 'some property of moving bodies"' (Harman 1995, p. 31).

In 1851, Maxwell wrote a letter to his friend Lewis Campbell asking, "Is truth nowhere but in Mathematics?" Maxwell’s own response was: "I am persuaded that the study of $x$ and $y$ is to men an essential preparation for the intelligent study of the material universe" (Maxwell 1990, vol. I, 1851, p. 208). A few years later, this belief gave way to certainty. In a letter to George Wilson, dated 4 January 1855 , he confessed with an air of excitement that "It is easy to see that the number of these sensations corresponds

8. For more information on this, see Schaffer 1997, esp. pp. 459-68. 
to what may be called in mathematical language the number of independent variables, of which sensible colour is a function" (Maxwell 1990, vol. I, 1851 , p. 268). There exists an analogy between the laws of numbers and the laws that govern the behavior and properties of physical objects. Maxwell made the notions of physical and mathematical analogy the cornerstones to the methodology he both preached and practiced. This methodology, for example, underpins his elegant mathematical expression of electromagnetism. In a manuscript of 1855, "On Faraday's Lines of Force," Maxwell termed his methodology "Physical Analogy." It is a methodology that

combines the advantages, while it gets rid of the disadvantages both of premature physical theories and technical mathematical formulæ. I mean the method of Physical Analogy. Of this we have instances in the substitution of numbers for quantities in all calculations, in the use of lines in mechanics to represent forces and velocities, in the partial analogy between the motion of light and that of a particle, and the more complete analogy between the motion of light and that of a vibration in an elastic medium. (Maxwell 1990, vol. I, 1855, p. 355)

In another draft of this paper, which he prepared for Transactions of the Cambridge Philosophical Society (Read on December 10, 1855 and February 11, 1856), Maxwell repeated the methodology's benefits and urgency in a more assertive tone, concluding that "the aim of exact science is to reduce the problems of nature to the determination of quantities by operations with numbers" (Maxwell 1864, pp. 27-8). And as he explained in 1860 in a lecture he delivered at King's College, London, scientific discovery involves more than mere mathematical descriptions of fundamental ideas: "in the study of Natural Philosophy we shall endeavour to put our calculations into such a form that every step may be capable of some physical interpretation, and thus we shall exercise powers far more useful than those of mere calculation- the application of principles, and the interpretation of results" (Maxwell [1860] 1990, p. 672). When we come to interpret physically the theorems of mathematical systems, we nevertheless "find that we can not only say "This is so' but "This must be so"” or that "This ought to be so, according to the analogy of nature," for "otherwise it would not be consistent with the first principles of truth" (Maxwell 1990, vol. I, 1860, p. 670). Naturally, our understanding of the properties and nature of atoms falls within the remit of "This must be so"” or "'This ought to be so," because "In studying the constitution of bodies we are forced from the very beginning to deal with particles which we cannot observe" (Maxwell 1875b, p. 357). But this does not pose any fatal problem to scientific progress. On the

9. "Physical analogy" is used here as a covering term; for a distinction between model and analogy, see Mellor 1968, esp. p. 282. 
contrary, he continues to advise that "if by the careful study of the laws of nature and their dependence on each other we have been trained into watchfulness over the processes of our own minds, and clear habits of thought, we shall come all the better prepared for the study of higher problems" (Maxwell 1990, vol. I, 1860, p. 668).

Maxwell's "physical analogy" has been the subject of rigorous critical scrutiny since at least 1955, when Joseph Turner's pioneering essay on the subject appeared (Turner 1955). A number of historians and philosophers of science followed suit, uncovering and shedding light on various aspects of Maxwell's methodology (i.e., Cat 2001; Kargon 1969; Wise 1982; Simpson 1997; Siegel 1991; Harman 1998, pp. 71-112). Mary Hesse has discerned four different types of theoretical method in Maxwell's thought. These are the hypothetical, the mathematical, the analogical and the deduction from experiments (Hesse 1973, p. 87). The hypothetical method deals with hypotheses that are entirely mechanical in character, viz, "no physical quantities are involved except those concerning masses in motion and the forces of weight, impact, pressure, and friction" (Hesse 1973, p. 88). Nevertheless, when it comes to a subject matter that is less obviously mechanical, Maxwell was very critical of this type of method, criticizing its pervasiveness in nineteenth-century physics. In these cases, physical hypotheses can only provide a partial explanation, making us liable to turn a blind eye to facts (Hesse 1973, p. 88). Maxwell was also critical of the purely mathematical method, the analytical subtleties of which often steer physicists into losing sight of the phenomena to be explained. Physical analogy, Hesse continues, is positioned midway between "the 'rash assumptions' of physical hypotheses and the 'analytical subtleties' of mathematical formulae" (Hesse 1973, p. 89). Within the parameters of this methodology, we may discern, on the one hand, formal analogies of equations that do not imply identity of physical substance or processes. These formal analogies serve as thinking aids in our understanding of formal relationships, enabling the "transfer of mathematical results from one system to another irrespective of subject matter" (Hesse 1973, p. 90). For example, a fluid flow may be compared to heat flow or electric induction, without nevertheless implying that these latter processes involve any kind of fluid in motion. On the other hand, there exist systems of ideas that are really analogous in form, "which must be distinguished from those that are merely mathematical, and when such analogies are found, they lead 'to a knowledge of both [systems], more profound than could be obtained by studying each system separately"' (Hesse 1973, p. 90). Here, although the causes of experimentally unobservable processes cannot be identified with their formal analogues in empirically observed systems, the relations between causes and effects are so similar that a method of representation so general is required as to express the "real similarity of relations without introducing unwarranted hypothetical ideas into the expression of 
the cause" (Hesse 1973, p. 90). These general methods are to be deduced from experiments, adopting an approach similar to the one Newton used to derive the law of gravitation. This is the deduction from experiments method, and as Hesse asserts, "it should be clear by now that the methods of analogy and of deduction from experiments are not separate methods but aspects of a single method" (Hesse 1973, p. 97). Giora Hon and Bernard Goldstein concur that Maxwell "insisted on avoiding, on the one hand, any 'favorite hypothesis' about the micro-level from which the macro-phenomena could be deduced and, on the other, any purely formal expression of remote 'analytical subtleties' without consideration of its physical interpretation" (Hon and Goldstein 2012, p. 253). And like Hesse, they have observed that Maxwell "sought a 'middle way' which he accomplished by means of analogy. Thus he took advantage of both phenomenalism and mathematical formalism" (Hon and Goldstein 2012, p. 253). This line of argument stretches at least as far back as Turner's essay. However, Hon and Goldstein have continued to stress that Maxwell "appealed to imaginary physics, that is, the analogue to electromagnetism is a system of an imaginary incompressible fluid, where 'imaginary' is not to be conflated with 'ideal'. Maxwell constructed an imaginary physical system, contrived solely for the purpose of developing a mathematical scheme applicable to a specific physical domain. He could then draw consequences from this imaginary system to the physical domain of electromagnetism that was rich in experimental results" (Hon and Goldstein 2012, p. 253). Within this framework, "Maxwell's innovation is to appeal to the power of imagination, and its range was not limited to known physical systems" (Hon and Goldstein 2012, p. 253). According to Hon and Goldstein, Maxwell referred to this methodology as "mathematical analogy" to distinguish it from Thomson's "physical analogy," but the term Maxwell chose, they contend, is unfortunate because it masks its originality and does little to help to distinguish it from the standard use of analogy (Hon and Goldstein 2012, p. 253). That said, Maxwell's "mathematical analogy," as defined by Hon and Goldstein, is akin to Hesse's account of Maxwell's "physical analogy," but the former place more emphasis on the imaginary systems that Maxwell contrived, "where 'imaginary' is not to be conflated with 'ideal"' (Hon and Goldstein 2012, p. 253). ${ }^{10}$ Jordi Cat, too, has shown that "contrary to recent emphasis on the

10. At this point, it should be noted that Maxwell's considerations about mathematics and physics have a resemblance to the idea of "pre-established harmony" between the two areas, which later played an important role in theoretical physics, and as Kragh has shown, it was an issue that preoccupied vortex physicists at the time of Maxwell. Maxwell appears to be anticipating, among others, David Hilbert and Hermann Minkowski, both of whom, Kragh has observed, promoted the notion of extending the range of pure mathematics to physics, maintaining at the same time "that in the end the validity of the axiomatic theory of the future had to depend on observed facts and that experiment must be the final arbiter of physical theory" (Kragh 2015, 520). 
nineteenth century rejection of imagination in science in favor of mechanical objectivity of instruments and rules, Maxwell's view pointed to an inclusive methodology that would both accommodate and harness the use of the imagination" (Cat 2001, p. 398).

Maxwell did not actually use the method of physical analogy to develop a theory of the behavior and nature of an atomic molecule's constituent components, but he never shied away from underlining its merits. In his comments on Kelvin's atomic theory, for instance, he famously wrote: "the method by which the motion of this fluid [vortex atom] is to be traced is pure mathematical analysis. The difficulties of this method are enormous, but the glory of surmounting them would be unique" (Maxwell 1875a, p. 45). Kelvin's vortex atoms can be observed and studied only mathematically, but mathematical analysis must be tied to phenomenalism and to theoretical interpretations of such phenomena as mass and force variations, gravitation and heat conduction. It is this principle that led Maxwell, as we have seen, to criticize Kelvin's (and Boscovich's) atomic theory. He also surmised that the ultimate atoms ought to be vibrationless particles that could be centers of force or material objects with a stated mass, the ability to exert attractive and repulsive forces, and possibly the ability to interpenetrate. To ensure the stability of conservative systems, moreover, ultimate atoms must remain in a confined state, for molecules, to recall Maxwell's words, "remain unbroken and unworn" (Maxwell 1873, p. 441), even as they must evidently have internal parts.

Composed in 1878 and entitled "A Paradoxical Ode After Shelley," Maxwell's last poem provides significant insight into his view on the ultimate atoms and the means by which we may study them. The poem opens with the following lines: "My soul's an amphicheiral knot / Upon a liquid vortex wrought / By Intellect in the Unseen residing" (Campbell and Garnett 1882, pp. 649-51). Maxwell's "liquid vortex" refers to Thomson's vortex atom theory, according to which, as we have seen, knotted vortices (atoms) in the ether comprise all chemical elements. The "amphicheiral knot" in the field of knot theory is a reference to an oriented knot that is equivalent to its mirror image. ${ }^{11}$ Coming from the Greek $\alpha \mu \varphi^{\prime} \chi \varepsilon \varepsilon \rho \alpha \varsigma$ ( $\alpha \mu \varphi^{\prime}$ [amphi] and $\chi \varepsilon^{\prime} i \rho$ [cheir-hand]), we may translate "Amphicheiral" as "ambidextrous," so that no matter whether the knot is left- or right-handed, it will be deformed to its mirror image. Maxwell's amphicheirally knotted soul is configured according to the choreographies of Thomson's vortex rings, which reside "in the Unseen" and can be conjured up only by means of the imagination. The "Unseen" is the first of several references to The Unseen Universe and its sequel

11. For more information on this point and an informative discussion of the poem more generally, see Silver 2008, pp. 1266-70. See also, Pratt-Smith 2014, pp. 254-56. 
Paradoxical Philosophy, an imagined dialogue between Christian scientists and fictitious German materialist Hermann Stoffkraft, to whom Maxwell's poem is addressed (Maxwell 1878, pp. 141-43). A left-handed trefoil knot adorns the top of The Unseen's title page. P. M. Harman reminds us that Stewart and Tait clearly intended their book as a refutation of John Tyndall's "Belfast Address" of 1874 (Heimann [sic] 1972, pp. 73-79), where the prominent Irish physicist and popularizer of science had famously expounded a materialistic conception of nature: "All religious theories, schemes and systems, which embrace notions of cosmogony, or which otherwise reach into the domain of science must, in so far as they do this, submit to the control of science, and relinquish all thought of controlling it" (Tyndall 1874, p. 61). In response, Tait and Steward employed the theories of Victorian physics (the ether, energy conservation, the second law of thermodynamics, and the vortex atom) to confute such "materialistic statements now-a-days so freely made" by demonstrating that "immortality is strictly in accordance with the principle of Continuity (rightly viewed)," that is, the principle of the uniformity of nature (Stewart and Tait 1875, p. xii). The Unseen Universe can thus be regarded, Harman observes, as a popularization of science for an ideological purpose, although it was intended as a contribution to the philosophy of nature (Heimann [sic] 1972, p. 73). Stoffkraft succumbs to the arguments of a blunt polemic and is eventually convinced to trade his erroneous convictions for belief in the doctrines of Unseen Universe. Maxwell is nevertheless keenly aware of the messiness and incomplete nature of the vortex atom theory in particular and of theoretical physics more generally. Thus, the poem's first stanza continues, "While thou dost like a convict sit / With marlinspike untwisting it / Only to find my knottiness abiding." In his conviction that the Unseen has solved the problem of the ontology of the particles in which Maxwell's soul subsists, Stoffkraft remains a "convict" of his own designs who will never find his way out of the labyrinthine "knottiness" he professes to have unraveled. Entrusting the doctrines of the Unseen, Stoffkraft remains lost in the tangled webs of Maxwell's "knottiness," a pun on "naughtiness" characteristic of Maxwell's generally seriocomic response to his friend and co-author of The Unseen Tait.

We may never be able to provide a scientific answer to the question of the origin of both ultimate atoms and atomic molecules, but as long as the notion of an intervening agent does not thwart our quest for answers, the chain of knowledge is likely to extend indefinitely:

O never may direct Creation

Break in upon my contemplation, Still may the causal chain, ascending,

Appear unbroken and unending,

And, where that chain is lost to sight 
Let viewless fancies guide my darkling flight

Through Æon-haunted worlds, in order infinite.

The existence of a creator does not signify, for Maxwell, the point where science ends. Rather, it is the point where science starts, where a causal chain begins that has no discernible end to it. The means by which Maxwell intends to navigate his way through the universe's "Æon-haunted worlds" is "viewless fancies," the powers of the imagination that open a window into the unseen. It is noteworthy that in an alternative draft of the poem, we read "atom-haunted worlds" in lieu of "Æon-haunted worlds" (Pratt-Smith 2014, p. 256), indicating that what Maxwell had in mind, in part at least, was the nature of the atom. But the central question that grows naturally out of these lines concerns the nature of Maxwell's imaginative flights. Stella Pratt-Smith, for one, concludes that Maxwell resorts in these final lines to the "calm surety of a prayer" that humbly admits the limitations of both empiricism and the imagination (Pratt-Smith 2014, p. 256). But Maxwell appears to be celebrating here the powers of the imagination and science, which may drive us some way closer to an appreciation of the perfection of the Creation. The fact that there is no end to the knowledge we can acquire about the physical world, along with the fact that empirical investigation does have limitations as "that chain is lost to sight," do not signify the limits of the imagination and science, but their boundless potential, which is a reflection of the unfathomable complexity of God's designs. ${ }^{12}$ Maxwell was very critical of the extravagant speculations that Tyndall's "Scientific Use of the Imagination" entails (i.e., Brown 2013, pp. 142-63). In Maxwell's seriocomic phrase, Tyndall's pronouncements may "Tyndalize the imagination" (Maxwell 1995, vol. II, 1871, p. 617), but they offer little more, in Daniel Brown's words, than a "solipsistic failure, whereby the authority attributed to the faculty serves to reify its fancies [...] rather than "land[ing] us on the solid shores of fact"' (Brown 2013, p. 156). Given Maxwell's aphorisms "of the siren song of the scientific imagination," even as late as in circa January 1879 (Maxwell $2002 \mathrm{~b}$, vol. III, 1879, pp. 745-46), it is unlikely that he would pray for his post-mortem imagination's unbridled freedom, which would render him a perennial captive, like Stoffkraft, of its siren songs. This, for him, would be a "curse" (Maxwell 2002b, vol. III, 1879, pp. 745-46). Rather, his reliance on the imagination in these final lines of the poem is informed by what Hon and Goldstein identified in Maxwell's thought as "mathematical analogy," which allows for the contrivance of an imaginary physical system expressed in the

12. This exposition of Maxwell's faith and his view on the science/religion issue lends support to Paul Theerman's thesis that "Maxwell denied that scientific truth was dependent on religious truth, or the reverse. Nonetheless, scientific conclusions could enrich religious contemplation of God's actions in nature" (Theerman 1986, p. 312). 
language of mathematics that is then applied to a specific physical domain, so that mathematical formalism and phenomenalism remain closely tied.

\section{Conclusion}

Throughout his career, Maxwell became ever more convinced of the atomic nature of matter, especially through his study of gases, without, however, reaching a final verdict on the nature of the ultimate building blocks of all physical reality. Meanwhile he toyed with several models in his attempts to account for the properties of gases (e.g., mutually repelling point atoms) and also discussed several others (e.g., Kelvin's vortex atom). Maxwell's studies suggested to him that physical bodies are made of different kinds of atomic molecules that exist in the form material bodies or energy points, exerting a field of attractive and repulsive forces. They have a particular mass and undergo continuous configurations defined by their motions. They are capable of vibration, emitting and absorbing light at varying frequencies, and they could very well be capable of occupying the same point in space. Due to their vibrational/rotational motions, atomic molecules must consist of ultimate atoms, vibrationless particles that could be centers of force or material objects with a stated mass, the ability to exert attractive and repulsive forces, and possibly the ability to interpenetrate. The indivisibility and indestructibility of atomic molecules as conservative systems means that ultimate atoms exist only in a confined state, never to be empirically observed as free particles. They can nevertheless be studied mathematically, and while we are not able to provide a scientific answer regarding the ultimate origin of their properties, we can, by means of physical analogy, study their relations and describe the laws that govern them.

\section{References}

Achinstein, Peter. 2007. “Atom's Empirical Eve: Methodological Disputes and How to Evaluate Them." Perspectives on Science 15: 359-390. DOI: https://doi.org/10.1162/posc.2007.15.3.359

Boscovich, Roger Joseph. 1766. Dissertatio De Lumine: Pars Secunda. Vindobonæ: Typis Joannis Tomæ.

Boscovich, Roger Joseph. (1758) 1966. A Theory of Natural Philosophy. Cambridge; MA: MIT Press.

Brown, Daniel. 2013. The Poetry of Victorian Scientists: Style, Science and Nonsense. Cambridge: Cambridge University Press. DOI: https://doi.org/10.1017 /CBO9781139151078

Brush, Stephen G. 2003. "Introduction." Pp. 1-33 in The Kinetic Theory of Gases: An Anthology of Classic Papers with Historical Commentary. London: Imperial College Press. DOI: https://doi.org/10.1142/9781848161337_0001 
Brush, Stephen. 1976. The Kind of Motion We Call Heat: A History of the Kinetic Theory of Gases in the Nineteenth Century, vol. 1: Physics and the Atomists. Amsterdam: North Holland.

Brush, Stephen, and Elizabeth Garber, eds. 1995. Maxwell on Heat and Statistical Mechanics. Bethlehem: LeHigh University Press.

Campbell, Lewis, and William Garnett. 1882. The Life of James Clerk Maxwell. London: Macmillan.

Cat, Jordi. 2001. "On Understanding: Maxwell on the Methods of Illustration and Scientific Metaphor." Studies in the History and Philosophy of Modern Physics 32: 395-441. DOI: https://doi.org/10.1016/S1355-2198(01) 00018-1

Darrigol, Olivier, and Jürgen Renn. 2013. “The Emergence of Statistical Mechanics." Pp. 765-788 in The Oxford Handbook of the History of Physics. Edited by Jed Z. Buchwald and Robert Fox. Oxford: Oxford University Press.

Dyson, Freeman. 1999. "Why is Maxwell's Theory So Hard to Understand?" In James Clerk Maxwell Commemorative Booklet, Fourth International Congress of Industrial and Applied Mathematics. Edinburgh.

Garber, Elizabeth. 1978. "Molecular Science in Late-Nineteenth-Century Britain." Historical Studies in the Pbysical Sciences 9: 265-297. DOI: https://doi.org/10.2307/27757380

Garber, Elizabeth. 2014. “Maxwell’s Kinetic Theory 1859-70.” Pp. 139-153 in James Clerk Maxwell: Perspectives on His Life. Edited by Raymond Flood, Mark McCartney, and Andrew Whitaker. Oxford: Oxford University Press.

Garber, Elizabeth, Stephen Brush and C. W. F. Everitt, eds. 1986. Maxwell on Molecules and Gases. Cambridge: Cambridge University Press.

Gell-Mann, Murray. 1997. "Quarks, Color, and QCD.” Pp. 625-633 in The Rise of the Standard Model: Particle Physics in the 1960s and 1970s. Edited by Lillian Hoddeson, Laurie Brown, Michael Riordan, and Max Dresden. Cambridge: Cambridge University Press. DOI: https:// doi.org/10.1017/CBO9780511471094.037

Harman, P. M. 1970. "Molecular Forces, Statistical Representation and Maxwell's Demon." Studies in History and Philosophy of Science Part A 1: 189-211. DOI: https://doi.org/10.1016/0039-3681(70)90009-9

Harman, P. M. 1972. “'The Unseen Universe': Physics and the Philosophy of Nature in Victorian Britain." The British Journal for the History of Science 6: 73-79. DOI: https://doi.org/10.1017/S0007087400011985

Harman, P. M. 1988. "Newton to Maxwell: The 'Principia' and British Physics." Notes E Records of the Royal Society of London 42: 75-96. DOI: https://doi.org/10.1098/rsnr.1988.0008

Harman, P. M. 1995. "Introduction." Pp. 1-38 in The Scientific Letters and Papers: Volume II: 1862-1873. Edited by P. M. Harman. Cambridge: Cambridge University Press. 
Harman, P. M. 1998. The Natural Philosophy of James Clerk Maxwell. Cambridge: Cambridge University Press.

Heilbron, J. L. 2015. "Boscovich in Britain." Pp. 99-116 in Relocating the History of Science: Essays in Honor of Kostas Gavroglu. Edited by Theodore Arabatzis, Jürgen Renn, and Ana Simões. New York: Springer.

Hesse, Mary. 1973. "Logic of Discovery in Maxwell's Electromagnetic Theory." Pp. 86-114 in Foundations of Scientific Method: The Nineteenth Century. Edited by Ronald N. Giere and Richard S. Westfall. Bloomington: Indiana University Press.

Holden, Thomas. 2004. The Arbitecture of Matter: Galileo to Kant. Oxford: Oxford University Press. DOI: https://doi.org/10.1093/0199263264.001.0001

Hon, Giora and Bernard R. Goldstein. 2012. "Maxwell's Contrived Analogy: An Early Version of the Methodology of Modeling." Studies in History and Philosophy of Modern Physics 43: 236-257. DOI: https://doi.org/10.1016 /j.shpsb.2012.07.001

Kargon, Robert. 1969. "Model and Analogy in Victorian Science: Maxwell's Critique of the French Physicists." Journal for the History of Ideas 30: 423-436. DOI: https://doi.org/10.2307/2708567

Knight, David M. 1967. Atoms and Elements: A Study of Theories of Matter in England in the Nineteenth Century. London: Hutchinson.

Kragh, Helge. 2003. "The vortex atom: A Victorian theory of everything." Centaurus 44: 32-114. DOI: https://doi.org/10.1034/j.1600-0498 .2002.440102.x

Kragh, Helge. 2015. "Mathematics and Physics: The Idea of a Pre-Established Harmony." Science E Education 24: 515-527. DOI: https://doi.org/10 .1007/s11191-014-9724-8

Levere, Trevor. 1971. Affinity and Matter. Oxford: Clarendon Press.

Marston, Philip L. 2007. "Maxwell and Creation: Acceptance, criticism, and his anonymous publication." American Journal of Physics 75: 731-740. DOI: https://doi.org/10.1119/1.2735631

Marston, Philip L. 2014. "Maxwell, Faith and Physics." Pp. 258-291 in James Clerk Maxwell: Perspectives on His Life and Work. Edited by Raymond Flood, Mark McCartney, and Andrew Whitaker. Oxford: Oxford University Press.

Maxwell, James Clerk. 1864. "III. On Faraday's Lines of Force." Transactions of the Cambridge Philosophical Society 10: 27-83.

Maxwell, James Clerk. 1867. "On the Dynamical Theory of Gases." Philosophical Transactions 157: 49-88. DOI: https://doi.org/10.1098/rstl.1867.0004

Maxwell, James Clerk. 1871a. Theory of Heat. London: Longmans.

Maxwell, James Clerk. 1871b. "Mathematics and Physics: Address by J. Clerk Maxwell." Pp. 1-9 in Report of the Fortieth Meeting of The British Association for the Advancement of Science Held at Liverpool in September 1870. London: John Murray. 
Maxwell, James Clerk. 1873. "Molecules." Nature 8: 437-441. DOI: https://doi.org/10.1038/008437a0

Maxwell, James Clerk. 1875a. “Atom.” Pp. 36-49 in Encyclopadia Britannica, Ninth Edition, vol. 3. Edinburgh: Adams and Charles Black.

Maxwell, James Clerk. 1875b. "On the Dynamical Evidence of the Molecular Constitution of Bodies." Nature 11: 357-359. DOI: https://doi.org/10 $.1038 / 011357 \mathrm{a} 0$

Maxwell, James Clerk. 1875c. "On the Dynamical Evidence of the Molecular Constitution of Bodies, II." Nature 11: 374-377. DOI: https:/doi.org/10 $.1038 / 011374 \mathrm{a} 0$

Maxwell, James Clerk. 1878. "Paradoxical Philosophy." Nature 19: 141-143. DOI: https://doi.org/10.1038/019141a0

Maxwell, James Clerk. 1990. The Scientific Letters and Papers of James Clerk Maxwell, Volume I: 1846-1862. Edited by P. M. Harman. Cambridge: Cambridge University Press.

Maxwell, James Clerk. 1995. The Scientific Letters and Papers of James Clerk Maxwell, Volume II: 1862-1873. Edited by P. M. Harman. Cambridge: Cambridge University Press.

Maxwell, James Clerk. 2002a. The Scientific Letters and Papers of James Clerk Maxwell: Volume III: 1874-1879: Part I: 1874-1876. Edited by P. M. Harman. Cambridge: Cambridge University Press.

Maxwell, James Clerk. 2002b. The Scientific Letters and Papers of James Clerk Maxwell, Volume III: 1874-1879, Part II: 1877-1879. Edited by P. M. Harman. Cambridge: Cambridge University Press.

Mellor, D. H. 1968. "Models and Analogies in Science." Isis 59: 282-290. DOI: https://doi.org/10.1086/350397

Nye, Mary Jo. 1976. "The Nineteenth-Century Atomic Debates and the Dilemma of an 'Indifferent Hypothesis." Studies in History and Philosophy of Science Part A 7: 245-268. DOI: https://doi.org/10.1016/0039-3681 (76)90007-8

Porter, Theodore M. 1981. "A Statistical Survey of Gases: Maxwell's Social Physics." Historical Studies in the Physical Sciences 12: 77-116. DOI: https://doi.org/10.2307/27757490

Porter, Theodore M. 1986. The Rise of Statistical Thinking 1820-1900. Princeton: Princeton University Press. DOI: https://doi.org/10.1515 19780691210520

Pratt-Smith, Stella. 2014. "Boundaries of Perception: James Clerk Maxwell's Poetry of Self, Senses and Science." Pp. 233-257 in James Clerk Maxwell: Perspectives on His Life and Work. Edited by Raymond Flood, Mark McCartney, and Andrew Whitaker. Oxford: Oxford University Press.

Pullman, Bernard. 1998. The Atom in the History of Human Thought. Translated by Axel Reisinger. Oxford: Oxford University Press. 
Rocke, Alan J. 1984. Chemical Atomism in the Nineteenth Century: From Dalton to Cannizzaro. Ohio: Ohio State University Press.

Schaffer, Simon. 1997. "Metrology, Metrication, and Victorian Values." Pp. 438-474 in Victorian Science in Context. Edited by Bernard Lightman. Chicago: Chicago University Press.

Scott, Wilson L. 1970. Conflict between Atomism and Conservation Theory: 1644-1860. New York: Elsevier.

Siegel, Daniel. 1991. Innovation in Maxwell's Electromagnetic Theory. Cambridge: Cambridge University Press.

Silver, Daniel S. 2008. "The Last Poem of James Clerk Maxwell." Notices of the AMS 55: 1266-1270.

Simpson, Thomas K. 1997. Maxwell on the Electromagnetic Field: A Guided Study. New Brunswick: Rutgers University Press.

Smith, Crosbie and M. Norton Wise. 1989. Energy and Empire: A Biographical study of Lord Kelvin. Cambridge: Cambridge University Press.

Stewart, Balfour and Peter Guthrie Tait. 1875. The Unseen Universe: Or, Physical Speculations on a Future State. London: Macmillan.

Theerman, Paul. 1986. "James Clerk Maxwell and Religion." American Journal of Physics 54: 312-317. DOI: https://doi.org/10.1119/1.14636

Turner, Joseph. 1955. "Maxwell on the Method of Physical Analogy." The British Journal for the Philosophy of Science 6: 226-238. DOI: https://doi .org/10.1093/bjps/VI.23.226

Tyndall, John. 1871. Fragments of Science for Unscientific People: A Series of Detached Essays, Lectures, and Reviews. New York: D. Appleton and Company. DOI: https://doi.org/10.5962/bhl.title.25271

Tyndall, John. 1874. Address Delivered Before the British Association Assembled at Belfast: with Additions. London: Longmans.

Wise, Norton M. 1982. "The Maxwell Literature and British Dynamical Theory." Historical Studies in the Physical Sciences 13: 175-205. DOI: https://doi.org/10.2307/27757511 